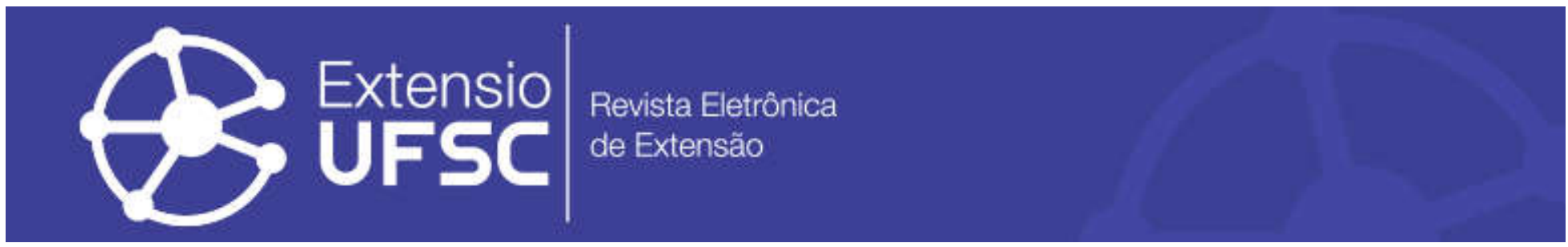

\title{
CONTRIBUIÇÕES DA EXTENSÃO UNIVERSITÁRIA COMO ESTRATÉGIA DE ASSISTÊNCIA NA PROMOÇÃO DA SEGURANÇA DO PACIENTE
}

\author{
Larissa de Oliveira Passamai \\ Universidade Estadual de Santa Cruz \\ spassamai@hotmail.com \\ Tércia Oliveira Coelho \\ Universidade Estadual do Sudoeste da Bahia \\ terciaoc@gmail.com \\ Gabriella Lins Guimarães Guirra de Carvalho \\ Universidade Estadual de Santa Cruz \\ gabriella.lins@yahoo.com.br
}

\author{
Priscila dos Santos Carvalho Chavenco \\ Universidade Católica de Salvador \\ priccihsl@gmail.com \\ Noélia Silva Oliveira \\ Universidade Estadual de Santa Cruz \\ nosilva@uesc.br
}

Sônia Maria Isabel Lopes Ferreira Universidade Estadual de Santa Cruz smilferreira@uesc.br

\section{Resumo}

Objetivo: relatar a experiência extensionista do Programa de Extensão Gestão do Cuidar em Saúde, da Universidade Estadual de Santa Cruz. Método: relato de experiência das ações desenvolvidas entre abril/2017 e setembro/2018, pelos componentes do Projeto de Extensão, e a Comissão de Controle de Infecção Hospitalar, na construção de um material educativo produzido com a finalidade de contribuir com a formação de uma Cultura de Segurança do Paciente na implantação do Núcleo de Segurança do Paciente em um hospital do Sul da Bahia. Resultados: inicialmente elaboraram-se oito cartilhas sobre as metas de segurança do paciente e, posteriormente, rodas de conversas com profissionais de saúde da rede hospitalar para disseminação do conhecimento e formação de grupos de trabalhos para a construção dos protocolos de segurança do paciente. Participaram da ação aproximadamente 100 profissionais de saúde. Conclusão: essa ação possibilitou a docentes e discentes atuarem de forma multidisciplinar, na implantação das estratégias de segurança do paciente, contribuindo para a redução de riscos decorrentes da assistência à saúde.

Palavras-Chave: Segurança do Paciente. Educação Permanente. Assistência em Saúde.

\section{CONTRIBUTIONS OF UNIVERSITY EXTENSION AS AN ASSISTENCE STRATEGY IN PROMOTING PATIENT SAFETY}

\begin{abstract}
Objective: to report the extensionist experience of Health Care Management Extension Program at the State University of Santa Cruz. Methods: an experience report was developed of the actions that took placebetween April/2017 and September/2018, by the components of the Extension Project and the Hospital Infection Control Commission, in the construction of an educational material produced in order to contribute to the formation of a Patient Safety Culture in the implementation of the Patient Safety Center in a hospital in the southern of Bahia. Results: initially, 8 booklets were elaborated on patient safety goals and, later, chat circles with health professionals to disseminate knowledge and form working groups for the construction of patient safety protocols. Approximately 100 health professionals participated in the action. Conclusion: this action enabled teachers and students to act in a multidisciplinary manner in the implementation of patient safety strategies, contributing to the reduction of risks arising from health care.

Keywords: Patient Safety. Permanent Education. Health Assistance.
\end{abstract}




\title{
CONTRIBUCIONES DE LA EXTENSIÓN UNIVERSITARIA COMO ESTRATEGIA DE ASISTENCIA EN LA PROMOCIÓN DE LA SEGURIDAD DEL PACIENTE
}

\begin{abstract}
Resumen
Objetivo: reportar la experiência extensionista del Programa de Extensión de Gestión del Cuidar en la Salud de la Universidad Estatal de Santa Cruz. Método: informe de experiencia de las acciones desarrolladas entre abril/2017 y septiembre/2018, por los componentes del Proyecto de Extensión, y la Comisión de Control de Infecciones Hospitalarias, en la construcción de un material educativo producido con el propósito de contribuir a la formación de una Cultura de Seguridad del Paciente en la implementación del Centro de Seguridad del Paciente en un hospital del sur de Bahía. Resultados: inicialmente, se prepararon ocho folletos sobre los objetivos de seguridad del paciente y, posteriormente, conversaciones con profesionales de la salud en la red del hospital para difundir el conocimiento y formar grupos de trabajo para la construcción de protocolos de seguridad del paciente. Aproximadamente 100 profesionales de la salud participaron en la acción. Conclusión: esta acción posibilitó a docentes y discentes actuar de forma multidisciplinaria, en la implantación de las estrategias de seguridad del paciente, contribuyendo a la reducción de riesgos derivados de la asistencia sanitaria.

Palabras clave: Seguridad del Paciente. Educación Permanente. Asistencia en Salud.
\end{abstract}




\section{INTRODUÇÃO}

A segurança do paciente pode ser definida como a redução do risco de danos desnecessários relacionados aos cuidados de saúde a um mínimo aceitável, segundo a Organização Mundial de Saúde (OMS, 2016). É um elemento essencial à qualidade da assistência à saúde, pois compreende ações que objetivam gerenciar e prevenir riscos aos quais os pacientes estão expostos (OMS, 2005). As falhas e fragilidades dessas ações proporcionam a ocorrência de eventos adversos (BRASIL, 2017).

Os eventos adversos (EA) são considerados incidentes que resultam em danos, não intencional e não relacionados à evolução natural da doença de base para um paciente (OMS 2016). O número de eventos adversos relacionados à assistência hospitalar correspondeu a $94 \%$ dos incidentes notificados, no ano de 2016. Sendo 53,2\% destes identificados no setor de internação. Quando comparado ao tipo de incidente, obtiveram-se 10.210 notificações relacionadas à lesão por pressão e 5.892 associados a quedas (BRASIL, 2017).

Maia et al. (2018) demonstrou que nos períodos de junho de 2014 a junho de 2016 foram registrados 63.933 notificações de eventos adversos relacionados a assistência à saúde. Destes, $417(0,6 \%)$ casos foram de óbitos. A maioria dessas notificações foi proveniente dos Núcleos de Segurança do Paciente dos hospitais (96,9\%). Por sua importância, tem se tornado há alguns anos um assunto prioritário nas instituições de saúde, levando a Organização Mundial de Saúde (OMS) a criar em 2004 a Aliança Mundial para a Segurança do Paciente, visando estabelecer conceitos e diretrizes relacionadas a este campo de saberes e práticas, bem como recomendar ações com o objetivo de reduzir riscos e eventos adversos.

A segurança do paciente compõe uma das dimensões avaliadas pelos indicadores de qualidade da assistência, auxiliando o gerenciamento de boas práticas em saúde e a tomada de decisões voltadas à assistência de qualidade e à segurança do paciente (BÁO et al., 2019; CIPRIANO; MEDEIROS; FATEL, 2014).

Desse modo, a segurança do paciente depende da adoção de estratégias que visam evitar a ocorrência de eventos adversos preveníveis e, quando não é possível, minimizar suas consequências para o paciente (Rede Brasileira de Enfermagem e Segurança do Paciente REBRAENSP, 2013). Dentre essas estratégias estão o gerenciamento de risco, a implantação de protocolos que subsidiem o planejamento da assistência, a comunicação efetiva entre as equipes e a adoção de uma cultura de segurança pelas instituições de saúde (OMS, 2005). 
Entende-se cultura de segurança como um dos valores da cultura organizacional cujo objetivo é favorecer ações coerentes e comportamentos mais adequados, tendo atitudes e normas como fundamentais para um ambiente seguro (HALLIGAN, ZECEVIC, 2011). A CSP é definida como o produto de valores, atitudes, percepções, competências e padrões de comportamento de grupos e de indivíduos (NIEVA, SORRA, 2003).

Buscando incentivar os serviços de saúde à adoção de práticas organizacionais para a assistência qualificada, o Brasil estabeleceu em 2013 a Política Nacional de Segurança do Paciente (PNSP), por meio da Portaria $\mathrm{N}^{\circ} 529$, de $1^{\circ}$ de abril de 2013, com o objetivo de alcançar melhores índices de qualidade da assistência, com foco na promoção da segurança do paciente e na satisfação do usuário dos serviços de saúde (BRASIL, 2013).

A PNSP instituiu a obrigatoriedade dos Núcleos de Segurança do Paciente (NSP) nas instituições públicas, privadas, filantrópicas, de ensino, entre outros, estabelecendo orientações para seu funcionamento. O NSP tem a responsabilidade de desenvolver o Plano de Segurança do Paciente (PSP), documentar estratégias e ações de gestão de risco, apropriar-se do seu papel como instância promotora de prevenção, controle e mitigação de eventos adversos. Uma das funções do NSP é promover uma Cultura de Segurança do Paciente (CSP) nos estabelecimentos de saúde imprescindível para a implantação e implementação de práticas assistências seguras ao paciente de forma sistematizada na instituição.

Diante do exposto, este estudo tem o objetivo de relatar a experiência extensionista do Programa de Extensão Gestão do Cuidar em Saúde, da Universidade Estadual de Santa Cruz (UESC)/Bahia, na implantação do Núcleo de Segurança do Paciente, em um hospital do Sul da Bahia/Brasil, através da construção de um material educativo produzido com a finalidade de contribuir com a formação de uma CSP institucional.

\section{METODOLOGIA}

Trata-se de um estudo descritivo, qualitativo, do tipo relato de experiência, do Programa de Extensão Gestão do Cuidar em Saúde da Universidade Estadual de Santa Cruz (UESC)/Bahia. E tem como objetivo desenvolver processos gerenciais, assistenciais e educacionais junto a pacientes com agravos clínicos e cirúrgicos, familiares, discentes e profissionais de saúde, nos serviços de saúde e domicílio. 
A atividade educativa foi realizada por duas enfermeiras mestrandas, dois discentes de enfermagem e duas docentes do curso de enfermagem da UESC que coordenam o projeto de extensão. Houve a parceria com a Comissão de Controle de Infecção Hospitalar (CCIH) de um hospital da região Sul da Bahia/Brasil, sendo o cenário das atividades desenvolvidas no período de abril de 2017 a setembro de 2018.

O público-alvo foram os profissionais de saúde atuantes na instituição hospitalar: 3 médicos, 77 enfermeiros, 3 nutricionistas, 2 fisioterapeutas, 3 farmacêuticos, 2 representantes da higienização e 10 funcionários da equipe administrativa, totalizando 97 profissionais.

As ações extensionistas foram realizadas a partir de um cronograma de atividades (quadro 1), garantindo o planejamento, a avaliação das tomadas de decisão e flexibilização de ações.

Quadro 1. Resumo do cronograma e das atividades realizadas pelo grupo extensionistas

\begin{tabular}{|c|c|c|c|}
\hline PERÍODO & ATIVIDADES DESENVOLVIDAS & $\begin{array}{l}\text { PÚBLICO- } \\
\text { ALVO }\end{array}$ & MEDIADORES \\
\hline Abril 2017 & $\begin{array}{c}\text { Discussão sobre o planejamento das estratégias } \\
\text { de elaboração das cartilhas educativas }\end{array}$ & $\begin{array}{l}\text { Enfermeiras da } \\
\text { CCIH }\end{array}$ & $\begin{array}{c}\text { Enfermeiras } \\
\text { Discentes } \\
\text { Docentes }\end{array}$ \\
\hline \multirow[t]{4}{*}{$\begin{array}{l}\text { Maio/2017 a } \\
\text { dezembro de } \\
2018\end{array}$} & $\begin{array}{l}\text { Estudos e levantamento do material para a } \\
\text { produção das cartilhas }\end{array}$ & $\begin{array}{l}\text { Enfermeiras da } \\
\text { CCIH }\end{array}$ & $\begin{array}{l}\text { Enfermeiras } \\
\text { Discentes } \\
\text { Docentes }\end{array}$ \\
\hline & $\begin{array}{c}\text { Articulação dos materiais coletados para a } \\
\text { produção da cartilha educativa e para o } \\
\text { protocolo de segurança do paciente }\end{array}$ & $\begin{array}{l}\text { Enfermeiras da } \\
\text { CCIH }\end{array}$ & $\begin{array}{c}\text { Enfermeiras } \\
\text { Discentes } \\
\text { Docentes } \\
\end{array}$ \\
\hline & Início da elaboração das cartilhas educativas & $\begin{array}{l}\text { Enfermeiras da } \\
\text { CCIH }\end{array}$ & $\begin{array}{c}\text { Enfermeiras } \\
\text { Discentes } \\
\text { Docentes }\end{array}$ \\
\hline & $\begin{array}{c}\text { Apresentação do esboço das cartilhas } \\
\text { educativas }\end{array}$ & $\begin{array}{l}\text { Enfermeiras da } \\
\text { CCIH } \\
\text { Docentes da } \\
\text { UESC }\end{array}$ & $\begin{array}{c}\text { Enfermeiras } \\
\text { Discentes }\end{array}$ \\
\hline Janeiro 2018 & $\begin{array}{l}\text { Adequação do material à necessidade do } \\
\text { serviço hospitalar }\end{array}$ & $\begin{array}{l}\text { Enfermeiras da } \\
\text { CCIH }\end{array}$ & $\begin{array}{c}\text { Enfermeiras } \\
\text { Discentes } \\
\text { Docentes }\end{array}$ \\
\hline Fevereiro 2018 & Apresentação das cartilhas educativas & $\begin{array}{l}\text { Enfermeiras da } \\
\text { CCIH }\end{array}$ & $\begin{array}{c}\text { Enfermeiras } \\
\text { Discentes } \\
\text { Docentes }\end{array}$ \\
\hline Março 2018 & $\begin{array}{c}\text { Ajustes finais na elaboração das cartilhas } \\
\text { educativas }\end{array}$ & $\begin{array}{l}\text { Enfermeiras da } \\
\text { CCIH }\end{array}$ & $\begin{array}{c}\text { Enfermeiras } \\
\text { Discentes } \\
\text { Docentes }\end{array}$ \\
\hline Abril 2018 & Revisão do conteúdo das cartilhas educativas & $\begin{array}{l}\text { Enfermeiras da } \\
\text { CCIH }\end{array}$ & $\begin{array}{l}\text { Enfermeiras } \\
\text { Discentes } \\
\text { Docentes }\end{array}$ \\
\hline Maio 2018 & Apresentação das cartilhas educativas & $\begin{array}{l}\text { Enfermeiras da } \\
\text { CCIH }\end{array}$ & $\begin{array}{c}\text { Enfermeiras } \\
\text { Discentes }\end{array}$ \\
\hline
\end{tabular}




\begin{tabular}{|c|c|c|c|}
\hline & & Docentes da & \\
\hline Junho 2018 & $\begin{array}{l}\text { Apresentação dos temas e proposta da } \\
\text { formação dos grupos de trabalho }\end{array}$ & $\begin{array}{c}\text { Profissionais da } \\
\text { equipe de saúde } \\
\text { do hospital }\end{array}$ & $\begin{array}{l}\text { Enfermeiras da } \\
\text { CCIH }\end{array}$ \\
\hline Julho 2018 & Rodas de conversa com as equipes de trabalho & $\begin{array}{c}\text { Profissionais da } \\
\text { equipe de saúde } \\
\text { do hospital }\end{array}$ & $\begin{array}{c}\text { Enfermeiras } \\
\text { Discentes } \\
\text { Docentes } \\
\text { Enfermeiras da } \\
\text { CCIH }\end{array}$ \\
\hline Agosto 2018 & $\begin{array}{l}\text { Construção dos protocolos de segurança do } \\
\text { paciente }\end{array}$ & $\begin{array}{c}\text { Enfermeiras } \\
\text { Discentes } \\
\text { Docentes da } \\
\text { Enfermeiras da } \\
\text { CCIH }\end{array}$ & $\begin{array}{c}\text { Profissionais da } \\
\text { equipe de saúde } \\
\text { do hospital }\end{array}$ \\
\hline Setembro 2018 & $\begin{array}{l}\text { Apresentação dos protocolos de segurança do } \\
\text { paciente }\end{array}$ & $\begin{array}{c}\text { Enfermeiras } \\
\text { Discentes } \\
\text { Docentes da } \\
\text { Enfermeiras da } \\
\text { CCIH }\end{array}$ & $\begin{array}{c}\text { Profissionais da } \\
\text { equipe de saúde } \\
\text { do hospital }\end{array}$ \\
\hline
\end{tabular}

Fonte: Dados das autoras (2020).

O cenário da atividade educativa foi um hospital que se constitui como uma instituição filantrópica, de grande porte, com 198 leitos, com atendimentos ao Sistema Único de Saúde (SUS), convênios e atendimentos particulares, com serviços de referência em clínica-médica, clínica-cirúrgica e alta complexidade em oncologia, traumatologia, cardiologia clínica e cirúrgica, hemodiálise e transplante renal e serve de campo de práticas e estágios aos cursos de enfermagem, medicina e biomedicina da UESC.

Para a o alcance dos objetivos, definiu-se e executou-se o seguinte plano de trabalho:

Passo 1: Levantamento teórico e pesquisa. Foram realizadas buscas em artigos científicos e em publicações da OMS sobre SP. Após este levantamento, decidiu-se adotar as recomendações do MS, a Portaria No 529, de $1^{\circ}$ de abril de 2013, a qual institui o PNSP, sendo que uma das atividades que as Instituições de saúde precisam cumprir são a elaboração e validação de protocolos e manuais voltados à SP.

Nesta fase, como referência acerca da discussão desta temática, tomamos as "Seis Metas Internacionais sobre a Segurança do Paciente" da OMS (2005), que são: identificação correta dos pacientes; comunicação efetiva, melhorar a segurança dos medicamentos de alta vigilância; cirurgia segura; redução do risco de infecções associadas aos cuidados em saúde e prevenção de danos decorrentes de quedas. As referidas metas compõem um passo extremamente importante para obter cuidado seguro, mas para alcançá-las, fazem-se necessárias mudanças na cultura organizacional da instituição hospitalar, voltadas para o aprendizado a partir de erros. 
Estas metas se constituem norteadoras para a implantação do NSP. Todos os trabalhadores em suas respectivas áreas de atuação tornam-se corresponsáveis na construção coletiva e individual do conhecimento e, mutuamente, devem ser auxiliados com o intuito de vigorar uma dinâmica de trabalho que possibilite a seguridade dos pacientes e de seus acompanhantes, não obstante, também dos envolvidos na prestação da assistência.

Passo 2: Processo de trabalho dos extensionistas. Cumprida a etapa anterior, iniciaram-se as atividades dos membros do programa de extensão, que foi a elaboração das cartilhas para a capacitação da equipe multiprofissional da instituição. Para o alcance desse objetivo foram elaboradas oito cartilhas, baseadas nas metas de segurança do paciente da OMS, assim denominadas: Cartilha 1: Cuidados Essenciais para Segurança do Paciente: Cultura de Segurança do Paciente; Cartilha 2: Cuidados Essenciais para Segurança do Paciente: Identificação do Paciente; Cartilha 3: Cuidados Essenciais para Segurança do Paciente: Cirurgia Segura; Cartilha 4: Cuidados Essenciais para Segurança do Paciente: Medicação; Cartilha 5: Cuidados Essenciais para Segurança do Paciente: Prevenção de Queda; Cartilha 6: Cuidados Essenciais para Segurança do Paciente: Prevenção de Lesão por Pressão; Cartilha 7: Cuidados Essenciais para Segurança do Paciente: Comunicação Efetiva e Cartilha 8: Cuidados Essenciais para Segurança do Paciente: Higiene das Mãos.

Passo 3: Formação de equipes de trabalho. Após a impressão das cartilhas, iniciou-se o processo de educação permanente às categorias profissionais, por meio de rodas de conversas, com base nas informações contidas nas cartilhas para a implantação do NSP. O processo educativo foi realizado a fim de fortalecer e consolidar as ações desenvolvidas pelo núcleo e reorganizar as práticas de saúde voltadas à SP.

As rodas de conversas foram realizadas em dez encontros, durante uma semana, previamente agendados, em local específico para este tipo de ação educativa. As atividades foram desenvolvidas no turno de trabalho dos profissionais, com 40 minutos cada, mediadas pelas docentes, discentes e enfermeiras do projeto de extensão.

As ações realizadas anteriormente foram o alicerce para a formação de uma cultura de segurança e construção dos Protocolos de Segurança do Paciente, a qual ocorreu a partir da divisão dos profissionais do hospital em grupos de trabalho, possibilitando a formação e o compartilhamento dos estudos desenvolvidos sobre as temáticas abordadas nas cartilhas. As discussões foram realizadas separadamente por equipe multidisciplinar, sendo formada por profissionais, Médicos, Enfermeiros, Nutricionistas, Fisioterapeutas, Farmacêuticos e representantes da Higienização do hospital. E posteriormente, com todos os grupos, o que 
permitiu a integração dos profissionais e possibilitou a construção coletiva do conhecimento e dos protocolos de segurança do paciente.

\section{RESULTADOS E DISCUSSÃO}

A Extensão na Educação Superior compõe uma das atividades preconizadas pelo Ministério da Educação, regulamentada pela Resolução N ${ }^{\circ}$ 7, de 18 de Dezembro 2018. Essas ações constituem um processo de desenvolvimento multiprofissional, interdisciplinar, políticosocial e técnico-científico que possibilitam a integração do ensino, serviço e comunidade através de pesquisas, construção de conhecimento e da implementação de intervenções realizadas junto à sociedade.

As ações extensionistas contribuem para a maior aproximação entre a universidade e a população. Neste aspecto, os participantes podem compartilhar experiências, permitindo a identificação das reais necessidades encontradas nos serviços e na comunidade. Oportunamente a sociedade civil tem acesso ao conhecimento de produção científica que permeia não apenas a academia, mas que por esta via de extensão alcança diferentes públicos e espaços.

A elaboração das cartilhas possibilitou integrar e atuar de forma multidisciplinar com os profissionais, além de possibilitar reflexões sobre a relevância e a necessidade dos serviços hospitalares em promover ações de saúde para os pacientes, familiares e acompanhantes, tendo em vista a assistência prestada ao paciente. Dessa forma, os conteúdos das cartilhas foram abordados da seguinte forma:

1- Cultura de Segurança do Paciente. A segurança do paciente constitui uma importante estratégia de promoção da saúde, prevenção de doenças e agravos e redução de riscos e/ou danos evitáveis causados aos pacientes. Esta cartilha introdutória tem a literatura convidativa para pacientes, acompanhantes e profissionais de saúde, de modo que haja ampla difusão do conhecimento sobre SP.

2- Identificação do Paciente. Compõe-se de estratégias para a implementação de ações voltadas à identificação do paciente e incluem, além da utilização de dispositivos e equipamentos, a incorporação e a priorização desta prática pela equipe multidisciplinar, assegurando-se de que a identificação do paciente será realizada na rotina hospitalar.

3- Higiene das Mãos. Abordou que as mãos podem transmitir microrganismos capazes de causar infecções, durante a assistência prestada, uma vez que a pele pode ser considerada um 
possível reservatório de microrganismos, sendo capaz de transmiti-los de forma direta (pele ou mucosa) ou indireta (objetos ou superfícies contaminados). A higiene das mãos é um dos procedimentos mais simples e mais eficazes na prevenção e controle da transmissão de doenças relacionadas à assistência à saúde. Visa à remoção dos microrganismos da flora residente e da flora transitória das mãos, além de células descamativas, pelo suor, sujidade e oleosidades. $\mathrm{O}$ objetivo principal desse processo é reduzir a transmissão de microrganismos, prevenindo as infecções, pois as mãos são o instrumento mais usado no cuidado do paciente (MAGNAGO, 2019).

4- Comunicação Efetiva. Permitiu discutir a promoção da SP, a qual possibilita o cuidado integral e contínuo desenvolvido a partir da transmissão de informações entre as pessoas. Sendo assim, os profissionais precisam ser capacitados e estar preparados para implementar estratégias que permitam construir relações estruturadas e sistematizadas, tendo em vista a qualidade da comunicação efetiva.

5- Prevenção de Lesão por Pressão. Constitui um indicador de avaliação da qualidade dos serviços e da segurança dos pacientes, o qual se compõe de ações essenciais às práticas de saúde, principalmente, no âmbito hospitalar.

6- Cirurgia Segura. Caracteriza-se pela implementação de práticas seguras voltadas à redução de riscos ao paciente antes, durante e após o tratamento cirúrgico. Essas práticas requerem ações que contribuam na prevenção, no monitoramento dos sinais e sintomas e na detecção de riscos a segurança do paciente no tratamento cirúrgico (ALPENDRE, 2017).

7- Prevenção de Quedas. Relaciona-se diretamente aos cuidados prestados ao paciente, principalmente quando estes são voltados a pessoas idosas, já que a assistência envolve aspectos culturais, conhecimentos e ações da equipe de saúde que possibilitem desenvolver habilidades teóricas e práticas, a fim de atender à necessidade dos usuários do serviço (OLIVEIRA, 2014).

8- Medicação Segura. A cartilha abordou os erros mais frequentes envolvendo administração de medicamentos, os quais foram classificados em erro de prescrição, de dispensação e de administração, que podem se associar a informações como sexo e idade do paciente, ao monitoramento, à condição clínica e à evolução da patologia (ANACLETO et al., 2010). Dessa forma, a promoção da segurança do paciente constitui uma prática essencial à prevenção e redução dos riscos envolvendo medicamentos nos serviços de saúde.

A Portaria No 3.390, de 30 de dezembro de 2013, que institui a Política Nacional de Atenção Hospitalar (PNHOSP) no âmbito do Sistema Único de Saúde (SUS), estabelecendo-se as diretrizes para a organização do componente hospitalar da Rede de Atenção à Saúde (RAS), 
define que o modelo de atenção hospitalar deve incluir estratégias de cuidado que assegure o acesso, a qualidade e a segurança do paciente.

Neste contexto, devem-se desenvolver ações que assegurem à qualidade da atenção e as boas práticas em saúde voltadas à segurança do paciente, com redução de incidentes desnecessários e evitáveis e atos inseguros relacionados ao cuidado (BRASIL, 2013).

A elaboração das cartilhas educativas sobre SP compreendeu uma importante ação extensionista para o processo de formação de uma CS e permitiu a exitosa implantação do NSP, além de contribuir para que os profissionais desenvolvam seu processo de trabalho regido por orientações que asseguram uma assistência voltada à SP, à prevenção de riscos e redução de danos correlacionados à assistência prestada.

A implantação dos protocolos de SP permite operacionalizar as normas e rotinas hospitalares, possibilitando a redução de eventos adversos relacionados à assistência e ao hospital. Desse modo, a interdisciplinaridade na construção das cartilhas e dos protocolos contribuiu para a discussão, reflexão e reorientação de práticas de cuidado em saúde e reorganização ambiente de trabalho.

O processo educativo nos serviços de saúde compõe-se de uma das estratégias utilizadas para a melhoria da qualidade da assistência e do aprimoramento e capacitação dos profissionais. Nesse sentido, possibilita o estímulo, motivação e autonomia do profissional, considerando as experiências e a valorização do conhecimento da equipe (SARDINHA et al., 2013). Dessa forma, a prática educativa desenvolvida a partir da elaboração das cartilhas sobre segurança do paciente permitiu a construção e o fortalecimento das relações entre as equipes do hospital e, consequentemente, com a universidade, partindo de um contexto intersetorial e interdisciplinar de discussão para o processo de formação de uma CSP.

As ações extensionistas voltadas à implementação do NSP convergem para o alinhamento da organização hospitalar com as metas da OMS sobre SP, evidencia práticas profissionais que podem ser melhor conduzidas para minimizar erros e potencializar uma nova e necessária modalidade de assistência cuja cultura busca a primazia da atualização em informações multiprofissional no sentido de aprimorar os serviços em saúde e alcançar o maior número de pessoas que fazem parte deste contexto (SOUZA, MENDES, 2019).

É importante destacar, como relata Rodrigues et al. (2013), que a extensão universitária proporciona a integração do ensino, pesquisa e serviços, tendo em vista as necessidades da equipe e da população atendida. Oliveira; Brêtas; Rosa, (2017), demonstraram que as ações extensionistas contribuíram para a formação cidadã dos profissionais, a partir das experiências e 
da identificação dos problemas locais, além de possibilitar a participação ativa e a autonomia dos extensionistas.

Levando-se em consideração a potencialidade dos projetos de extensão no serviço à comunidade e assistência em saúde, já se observa que a implementação de ações nesta vertente colabora para a efetivação de estratégias que vigoram na promoção de saúde de pessoas nas mais diferentes faixas etárias e ocupações na sociedade. Deste modo, a propagação de informações científicas por meio de palestras, roda de conversa, infográficos e material digital possibilitam envolver colaboradores de distintas áreas de atuação e esfera governamental (SILVA et al., 2019).

Desse modo, as atividades desenvolvidas no Programa de Extensão Gestão do Cuidar em Saúde da UESC possibilitaram aos extensionistas compartilhar conhecimentos produzidos e auxiliaram no desenvolvimento de habilidades voltadas à formação profissional e à qualidade da assistência prestada à comunidade.

A educação permanente em saúde consiste em uma importante estratégia de ensino utilizada para promover a discussão de temas essenciais ao exercício profissional, como a SP. Isto possibilita a formação de profissionais críticos, reflexivos e ativos nos ambientes de trabalho, o que permite reorientar práticas e reorganizar serviços de saúde voltados à assistência segura, adequada e de qualidade ao paciente (WEGNER et al., 2016).

Estudos identificaram que a educação voltada à melhora da assistência em saúde tem contribuído de forma significativa na atuação da equipe de enfermagem. Além de abordarem a necessidade e a importância da continuidade dessas atividades educativas para os profissionais que exercem ações relacionadas à SP (CARVALHO; ASSUNÇÃO; BOCCHI, 2019).

A construção da cultura de segurança constitui um desafio às instituições hospitalares, uma vez que requer tempo para a assimilação e implementação de novas práticas em saúde, voltadas à SP e à sensibilização dos profissionais da equipe de saúde, a fim de promover a conscientização relacionada à responsabilidade coletiva e compartilhada (WEGNER et al., 2016).

Desse modo, as ações extensionistas permitiram desenvolver importantes debates para a construção da cultura de segurança como tema a ser apreendido e praticado pelas diversas categorias profissionais, além de promover a institucionalização de melhores práticas nos ambientes de cuidados ao paciente.

\section{CONSIDERAÇÕES FINAIS}


Compreende-se, portanto, que as ações voltadas à SP se constituem essenciais para a prevenção de riscos e redução de incidentes associados à assistência voltada ao paciente. Deste modo, a consolidação das ações extensionistas realizadas com a equipe multidisciplinar na implantação do NSP e construção de protocolos contribuíram para a implementação dessas práticas, as quais são sugestíveis de continuidade para proporcionar um ambiente onde se enfatiza e prioriza a CSP como parte fundamental da dinâmica do serviço e da assistência de qualidade aos pacientes.

Tem-se, portanto, o trabalho desenvolvido pela equipe extensionista uma estratégia exitosa na confecção e distribuição das cartilhas, na construção coletiva de conhecimento e fomento na educação permanente, a partir das necessidades apresentadas pelo hospital. Logo, auxiliou na contribuição social da Universidade e no desenvolvimento de profissionais com consciência cidadã.

As ações neste campo extensionista proporcionaram a todos os envolvidos compreender a relevância e a necessidade da universidade e do hospital em promover boas práticas de saúde para os pacientes, por meio da implantação das estratégias de SP, visando reduzir, ao mínimo possível, os riscos desnecessários decorrentes da assistência à saúde. Conforme demonstrado para que as organizações hospitalares obtenham sucesso durante o processo de implantação de tais estratégias, é essencial o fortalecimento de uma cultura de segurança, seguindo as metas internacionais de segurança do paciente. Sendo assim, podem inferir que a estratégia utilizada de educar para construir foi assertiva para essa ação.

\section{REFERÊNCIA}

ALPENDRE, Francine Taporosky et al. Cirurgia segura: validação de checklist pré e pósoperatório. Rev. Latino-Am. Enfermagem, Ribeirão Preto, v. 25, 2017. Disponível em: http://www.scielo.br/scielo.php?script=sci_arttext\&pid=S010411692017000100357\&lng=en\&nrm=iso. Acesso em 31. out. 2019.

ANACLETO, Tânia Azevedo et al. ERROS DE MEDICAÇÃO. FARMACOVIGILÂNCIA HOSPITALAR: Como implantar. Pharmacia Brasileira, janeiro/fevereiro, 2010. Disponível em: http://www.cff.org.br/sistemas/geral/revista/pdf/124/encarte_farmaciahospitalar.pdf. Acesso em 09. set. 2019.

BÁO, Ana Cristina Pretto et al. Indicadores de qualidade: ferramentas para o gerenciamento de boas práticas em saúde. Rev Bras Enferm. v 72, n.2, p. 377-84, 2019. Disponível em: 
https://www.scielo.br/pdf/reben/v72n2/pt_0034-7167-reben-72-02-0360.pdf. Acesso em: 25 jun 2020 .

BRASIL. Gerência de Vigilância e Monitoramento em Serviços de Saúde (GVIMS). Gerência Geral de Tecnologia em Serviços de Saúde (GGTES). Agência Nacional de Vigilância Sanitária. Série Segurança do Paciente e Qualidade em Serviços de Saúde. Gestão de Riscos e Investigação de Eventos Adversos Relacionados à Assistência à Saúde. Brasília: Anvisa, 2017, p.17. Disponível:http://portal.anvisa.gov.br/documents/33852/3507912/Caderno+7+-

+ Gest $\% \mathrm{C} 3 \% \mathrm{~A} 3 \mathrm{o}+\mathrm{de}+$ Riscos + e+Investiga $\% \mathrm{C} 3 \% \mathrm{~A} 7 \% \mathrm{C} 3 \% \mathrm{~A} 3 \mathrm{o}+\mathrm{de}+$ Eventos + Adversos + Relacionados $+\% \mathrm{C} 3 \% \mathrm{~A} 0+$ Assist $\% \mathrm{C} 3 \% \mathrm{AAncia}+\% \mathrm{C} 3 \% \mathrm{~A} 0+\mathrm{Sa} \% \mathrm{C} 3 \% \mathrm{BAde} / 6$ fa4fa91-c652-4b8b-b56e-fe466616bd57.

Acesso em: 28 jun 2020.

BRASIL. Agência Nacional de Vigilância Sanitária. Resolução - RDC no 36, de 25 de julho de 2013. Institui ações para a segurança do paciente em serviços de saúde e dá outras providências. Diário Oficial da União [da] República Federativa do Brasil. 2013 Jul 26; 150(143 Seção 1):32-3.

BRASIL. Ministério da Saúde. Portaria No 3.390, de 30 de dezembro de 2013. Institui a Política Nacional de Atenção Hospitalar (PNHOSP) no âmbito do Sistema Único de Saúde (SUS), estabelecendo-se as diretrizes para a organização do componente hospitalar da Rede de Atenção à Saúde (RAS). Diário Oficial da União [da] República Federativa do Brasil. 2013 dez 30.

BRASIL. Gerência de Vigilância e Monitoramento em Serviços de Saúde- GVIMS.Gerência Geral de Tecnologia em Serviços de Saúde- GGTES. Agência Nacional de Vigilância SanitáriaANVISA. Boletim Segurança do Paciente e Qualidade em Serviços de Saúde no 15: Incidentes Relacionados à Assistência à Saúde- 2016. Brasilia, p. 1-20, 28 dez 2017.

BRASIL. Ministério da Educação. Conselho Nacional de Educação Câmara de Educação Superior. Resolução No 7, DE 18 DE DEZEMBRO DE 2018. Estabelece as Diretrizes para a Extensão na Educação Superior Brasileira e dá outras providências. BRASILÍA, 2018.

CARVALHO, Cesar Junior Aparecido de et al. Estratégias para segurança do paciente através da educação continuada no centro cirúrgico: um relato de experiência. Xviii sedu - semana da educação i congresso internacional de educação contextos educacionais: formação, linguagens e desafios. http://www.uel.br/eventos/semanadaeducacao/pages/arquivos/Anais/2019/EIXO\%206/3.\%2 0ESTRATEGIAS $\% 20$ PARA $\% 20$ SEGURANCA $\% 20$ DO $\% 20$ PACIENTE $\% 20 A T R A V E S \% 20$ DA $\% 20$ EDUCACAO $\% 20$ CONTINUADA $\% 20$ NO $\% 20$ CENTRO $\% 20$ CIRURGICO $\% 20 \mathrm{rel} . \mathrm{pd}$ f. Acesso em: 28 jun 2020.

CIPRIANO, Sonia Lucena; MEDEIROS, Adriane Lopes; FATEL, Karina de Oliveira. Instituto para Práticas Seguras no Uso de Medicamentos. SEGURANÇA DO PACIENTE GERENCIAMENTO DE RISCO E GESTÃO DE QUALIDADE. HOSP FASCICULO 3 WEB EDUC 2014. Disponível: https://www.eurofarma.com.br/wpcontent/uploads/2018/01/seguranca-paciente-gerenciamento-risco-gestao-qualidade.pdf. Acesso em: 28 jun 2020.

HALLIGAN, Michelle; ZECEVIC, Aleksandra. Safety culture in health care: a review of concepts, dimensions, measures and progress. BMJ Qual Saf. v. 20, n. 4, p. 338-43, 2011. 
LIMA JÚNIOR, F. A.; PANTOJA, M. DE S.; LIMA, K. V. M.; BORGES, R. M.; OLIVEIRA, A. S. DE; CHAVES, A. S. C.; BARROSO, R. B.; SILVA, V. C. DA. Implantação do núcleo de segurança do paciente: ações de capacitação e desenvolvimento institucional. Revista Eletrônica Acervo Saúde, v. 11, n. 8, p. e548, 19 mar. 2019.

MAGNAGO, Tânia Solange Bosi de Souza et al. Infraestrutura para higienização das mãos em um hospital universitário. Rev. Gaúcha Enferm. Porto Alegre, v. 40, n. spe, e20180193, 2019.

MAIA, Christiane Santiago et al. Notificações de eventos adversos relacionados com a assistência à saúde que levaram a óbitos no Brasil, 2014-2016. Epidemiol. Serv. Saude, Brasília,v. 27, n.2, p. e2017320, 2018.

NIEVA VF, SORRA J. Safety culture assessment: A tool for improving patient safety in health care organizations. Qual Saf Health Care 2003; 12(Supl. 2):ii17-23.

OLIVEIRA, Camila da Silva.; BRÊTAS, Ana Cristina Passarella.; ROSA, Anderson da Silva. A IMPORTÂNCIA DA EXTENSÃO UNIVERSITÁRIA NA GRADUAÇÃO E PRÁTICA PROFISSIONAL DE ENFERMEIROS. Currículo sem Fronteiras, v. 17, n. 1, p. 171-186, jan./abr. 2017.

OLIVEIRA, Danilo Ulisses de. AVALIAÇÃO DE QUEDAS EM IDOSOS HOSPITALIZADOS. 2014. 79 p. Dissertação de mestrado, Universidade Federal de Minas Gerais, Belo Horizonte, 2014.2 Disponível em: http://www.bibliotecadigital.ufmg.br/dspace/bitstream/handle/1843/ANDO-

9XRH2L/danilo_ulisses_de_oliveira.pdf?sequence=1. Acesso em: 25 jul 2017.

ORGANIZAÇÃO MUNDIAL DA SAÚDE (CH) [nternet]. Geneva: WHO; c2009-2016 [cited 2016 Dec 02]. Patientsafety: aboutus; [about 2 screens]. Disponível em: http:// www.who.int/patientsafety/about/en/. Acesso em: 28 jun 2020.

ORGANIZAÇÃO MUNDIAL DA SAÚDE (CH). World Alliance for PatientSafety WHO draft guidelines for adverse eventreporting andlearning systems: frominformationtoaction. Geneva: WHO, 2005.

ORGANIZAÇÃO MUNDIAL DA SAÚDE (CH). World Alliance for PatientSafety. Global patientsafetychallenge 2005-2006: clean careis safe care; 2005 [cited 2018 Jul 20]. Availablefrom: http://www.who.int/patientsafety/events/05/GPSC_Launch_ ENGLISH_FINAL.pdfREDE BRASILEIRA DE ENFERMAGEM E SEGURANÇA DO PACIENTE. Estratégias para a segurança do paciente: manual para profissionais da saúde. Porto Alegre (RS): EDIPUCRS, 2013 [citado 2016 dez 02]. Disponível em: http://www.rebraensp. com.br/pdf/manual_seguranca_paciente.pdf. Acesso em: 28 jun 2020.

RODRIGUES, Andréia Lilian Lima et al. CONTRIBUIÇÕES DA EXTENSÃO UNIVERSITÁRIA NA SOCIEDADE. Cadernos de Graduação - Ciências Humanas e Sociais. Aracaju, v. 1, n.16, p. 141-148, mar. 2013.

SARDINHA, Letícia Peixoto et al. Educação permanente, continuada e em serviço: desvendando seus conceitos. Enfermería Global. n. 29, p. 324-340, enero 2013. 
SILVA, T.; PIMENTA, S. R.; AMARAL, I.; RAMOS, T.; SOUZA, T.; PEREZ, A. P. O impacto das ações desenvolvidas pelo projeto de extensão ' $O$ toque pela vida' no aumento das mamografias realizadas pela Saúde Pública de Jataí, GO. Revista Brasileira de Extensão Universitária, v. 10, n. 2, p. 79-85, 1 ago. 2019.

SOUSA, Paulo; MENDES, Walter. Segurança do paciente: criando organizações de saúde seguras. 2nd ed. rev. Updt. Editora FIOCRUZ, 2019. 268 p.

WEGNER, Wiliam. Educação para cultura da segurança do paciente: Implicações para a formação profissional. Escola Anna Nery, v. 20, n.3. Jul-Set 2016.

Recebido em: 15/11/2019

Aceito em: 20/07/2020 